\title{
STABILITY PROPERTIES OF THE COLORED JONES POLYNOMIAL
}

\author{
CHRISTINE RUEY SHAN LEE
}

\begin{abstract}
It is known that the colored Jones polynomial of a +-adequate link has a well-defined tail consisting of stable coefficients, and that the coefficients of the tail carry geometric and topological information on the +-adequate link complement. We show that a power series similar to the tail of the colored Jones polynomial for +-adequate links can be defined for all links, and that it is trivial if and only if the link is non +-adequate.
\end{abstract}

Keywords. colored Jones polynomial, adequate link, stability, tail.

\section{INTRODUCTION}

For an oriented link $K \subset S^{3}$, the colored Jones polynomial is a sequence of Laurent polynomials $\left\{J_{K}^{n}(q)\right\}_{n=2}^{\infty}$, where $J_{K}^{n}(q) \in \mathbb{Z}\left[q^{-1 / 2}, q^{1 / 2}\right]$ is an invariant of $K$ for each $n$. In particular, $J_{K}^{2}(q)$ is the ordinary Jones polynomial. Unlike the Alexander polynomial, which has a definition based on the topology of the link complement, the colored Jones polynomial originated from representation theory and the ideas of quantum physics, with a definition that makes no explicit reference to the topology of the link. Thus, understanding the geometric and topological information that the polynomial carries has been a fundamental goal in the study of knots, 3-manifolds, and quantum invariants.

The colored Jones polynomial of a link can be defined and studied in terms of combinatorial properties of link diagrams. One approach to this is through the Kauffman bracket Kau87. Studying the combinatorics of the Kauffman bracket, Lickorish and Thistlethwaite [LT88] showed that the extreme degrees of the Jones polynomial for a link are bounded by concrete data from any diagram. If the link is semi-adequate, which means that it is +-adequate or --adequate, see Definition 2.3 and 2.4, then the bounds are sharp. The colored Jones polynomial of a semiadequate link has since been studied considerably [DL06, Sto11, Arm13, GL15, AD17, GNV16, GV17, $\mathrm{AD}$, and it has been shown to relate to the topology of essential surfaces and the geometry of the hyperbolic link complement [Kas97, MM01, MMO ${ }^{+} 02$, FGL02, GL05, DL07, FKP08, Gar11, FKP13, FKP14.

The coefficients of the colored Jones polynomial exhibit a stability behavior for semi-adequate links. Let $d(n)$ denote the minimum degree of $J_{K}^{n}(q)$. For $i \geq 2$, let $\beta_{i}$ be the coefficient of $q^{d(i)+(i-2)}$ of $J_{K}^{i}(q)$. Armond [Arm13] and Garoufalidis and Le [GL15] have independently shown that for all $n \geq i$, the coefficient of $q^{d(n)+(i-2)}$ of $J_{K}^{n}(q)$ is equal to $\beta_{i}$ if $K$ is +-adequate. That is, the first $n-1$ coefficients of $J_{K}^{n}(q)$ from $d(n)$ are stable for a link with a +-adequate diagram $D$, extending the results by Dasbach and Lin [DL06] on the first, second, and third coefficient of the colored Jones polynomial of adequate links. For a + -adequate link $K$, Armond and Garoufalidis and Le define a power series, called the tail of the colored Jones polynomial

$$
T_{K}(q)=\sum_{i=2}^{\infty} \beta_{i} q^{i},
$$

consisting of stable coefficients. These stable coefficients have been shown by the work of Futer, Kalfagianni, and Purcell to carry information on the geometric structure of the complement of a

Lee was partially supported by NSF grant MSPRF-DMS 1502860. 
+-adequate knot, and to provide sharp volume bounds on the complement of hyperbolic adequate links. See [FKP13] for the results and a detailed survey.

Rozansky has shown that stability behavior also occurs in the categorification of the colored Jones polynomial [FKS06, CK12, Roz14a], where a bi-graded chain complex $C^{K h}(K, n)$ is assigned to a link $K$, such that the graded Euler characteristic of the homology groups $H^{K h}(K, n)$ is the $n$th colored Jones polynomial $J_{K}^{n}(q)$. In [Roz14b], he shows that for $K$ a + -adequate link, one can define a family of maps

$$
f_{n}: \widetilde{H}^{K h}(K, n) \rightarrow \widetilde{H}^{K h}(K, n+1),
$$

such that $f_{n}$ is an isomorphism on $\widetilde{H}_{i, *}^{K h}$ for $i \leq n-1$, where $i$ is the homological grading. The tilde indicates the appropriate degree shift on the chain complex $C^{K h}(K, n)$, so that $f_{n}$ is a degreepreserving map for each $n$. In other words, for all $n \geq i$, homology groups $\widetilde{H}^{K h}(K, n)$ of homological grading less than $i-1$ are isomorphic to the homology groups $\widetilde{H}^{K h}(K, i)$ of homological grading less than $i-1$. As a result, he defines a tail homology of the colored Jones polynomial which is the direct limit of the direct system of $\left\{\widetilde{H}^{K h}(K, n)\right\}_{n=2}^{\infty}$ determined by $\left\{f_{n}\right\}$. The tail homology contains the stable homology groups of $C^{K h}(K, n)$ as $n$ increases. Therefore, we have a version of the results by Armond [Arm13] and Garoufalidis and Le [GL15] for the categorification of the colored Jones polynomial.

It is natural to ask whether similar results can be obtained outside the class of semi-adequate links. In particular, we consider $h_{n}(D)$, a lower bound for $d(n)$ from any diagram $D$ of the link, see (6). For a +-adequate link, we have that $d(n)=h_{n}(D)$, where $D$ is a +-adequate diagram [LT88, FKP11. See Corollary 2.11 for a proof of this result. If a link does not admit a +-adequate diagram, we may ask how $d(n)$ behaves with respect to $h_{n}(D)$. In this paper, we study the effect that a diagram $D$ being non +-adequate has on the difference $d(n)-h_{n}(D)$. We work with the unreduced version of the polynomial with the normalization convention as specified by Definition 2.2. Kalfagianni and the author have shown that $d(n)>h_{n}(D)$ for $n>2$ if $D$ is not +-adequate KL14]. Therefore, the colored Jones polynomial can characterize +-adequate links. Our main result, which extends that of [KL14], is the following.

Theorem 1.1. Let $D$ be a diagram of a link $K \subset S^{3}$ and for $n \geq 2$, let $d(n)$ be the minimum degree in $q$ of the $n$th colored Jones polynomial $J_{K}^{n}(q)$, with $h_{n}(D)$ the lower bound of $d(n)$ from $D$ as defined by (6). If $D$ is not +-adequate, then $d(n) \geq h_{n}(D)+n-2$ for $n>2$.

If $K$ is not +-adequate, then any diagram $D$ of $K$ is not +-adequate. Let $h_{n}$ be the maximum of $h_{n}(D)$ taken over all diagrams $D$ of $K$. This is a link invariant and by Theorem $1.1, d(n) \geq h_{n}+n-2$ for $n>2$. We use this to obtain a link invariant in the form of a power series $J_{K}^{+}(q)$, which vanishes if $K$ is not +-adequate as in [KL14]. This allows us to extend the construction of a tail of a +adequate link to all links. See the discussion in Section 4. We remark that while $J_{K}^{+}(q)$ coincides with $T_{K}(q)$ when $K$ is adequate, it is not the same as the "tail" considered in DL06, Arm13, since they consider coefficients from the actual minimum degree $d(n)$ of the polynomial.

In terms of the characterization of semi-adequacy by the colored Jones polynomial, note that Manchon [Man04] has constructed an infinite family of non +-adequate knots with diagrams $D$ for which $d(2)=h_{2}(D)$. An example of such a knot is $12 \mathrm{n} 706$, see KnotInfo [JCC14] for a diagram of the knot where $d(2)=h_{2}(D)=-4$. However, this knot is not +-adequate since the first coefficient of its Jones polynomial is 2, and it is known that for a +-adequate link, the first coefficient of its Jones polynomial is \pm 1 [DL06]. Manchon's examples show that the degree of the Jones polynomial is not enough to characterize links which are not +-adequate, while Kalfagianni [Kal18] has obtained a characterization of adequate links by the colored Jones polynomial using Theorem 1.1.

A conjecture made by Rozansky [Roz14b, Conjecture 2.13] stating that the tail homology he has constructed vanishes for non +-adequate links has now been proven [Lee18]. However, its 
direct implication for the colored Jones polynomial is weaker than Theorem 1.1, as it states that $d(n)-h_{n}(D) \geq f(n)$ where $f(n)=a n+b$ with constants $a<1, b$.

Since a diagram is --adequate if its mirror image is +-adequate, for the rest of the paper we will only deal with +-adequacy. For the results discussed for a +-adequate link, analogous statements for the maximum degree of the colored Jones polynomial of a --adequate link may be obtained by taking the mirror image $\bar{D}$ of a +-adequate diagram $D$, using the fact that $J_{\bar{D}}^{n}(q)=J_{D}^{n}\left(q^{-1}\right)$.

\section{Preliminaries}

2.1. Skein theory and the colored Jones polynomial. We follow the approach of [Lic97]. Let $F$ be an orientable surface which has a finite (possibly empty) collection of points $P$ specified on $\partial F$ if $\partial F \neq \emptyset$. A link diagram on $F$ consists of finitely many arcs and closed curves on $F$ such that

- There are finitely many transverse crossings with an over-strand and an under-strand.

- The endpoints of the arcs lie in $P$.

Two link diagrams on $F$ are isotopic if they differ by a homeomorphism of $F$ isotopic to the identity. The isotopy is required to fix $\partial F$.

Definition 2.1. Let $A$ be a fixed complex number. The linear skein $\mathcal{S}(F)$ of $F$ is the vector space of formal linear sums over $\mathbb{C}$ of isotopy classes of link diagrams $D$ in $F$ quotiented by the relations

(i) $D \sqcup \bigcirc=\left(-A^{-2}-A^{2}\right) D$, and

(ii) $Y / K=A)\left(+A^{-1} \stackrel{A^{2}}{\sim}\right.$.

We consider the linear skein $\mathcal{S}\left(\mathcal{D}^{2}, n\right)$ of the disc $\mathcal{D}^{2}$ with $2 n$-points specified on its boundary, where the boundary is viewed as a rectangle with $n$ marked points above and below, see Figure 1 for an example. For $D_{1}, D_{2} \in \mathcal{S}\left(\mathcal{D}^{2}, n\right)$, there is a natural multiplication operation $D_{1} \cdot D_{2}$ defined by identifying the top boundary of $D_{1}$ with the bottom boundary of $D_{2}$. This makes $\mathcal{S}\left(\mathcal{D}^{2}, n\right)$ into an algebra $T L_{n}$, called the $n$th Temperley-Lieb algebra. The algebra $T L_{n}$ is generated by a basis $\left.\right|_{n}, e_{n}^{1}, \ldots, e_{n}^{n-1}$, where $\left.\right|_{n}$ is the identity with respect to the multiplication and $e_{n}^{i}$ is a crossing-less link diagram as specified below in Figure 1 .
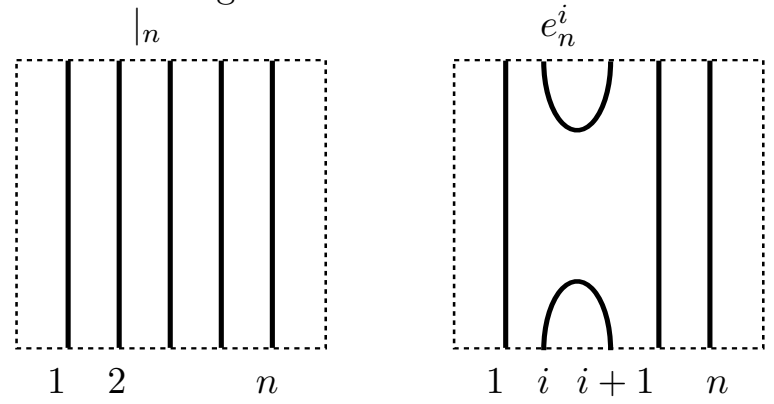

Figure 1. An example of the identity element $\left.\right|_{n}$ and a generator $e_{n}^{i}$ of $T L_{n}$ for $n=5$ and $i=2$.

We will use a shorthand notation which denotes $n$ parallel strands by $\left.\right|_{n}$.

Suppose that $A^{4}$ is not a $k$ th root of unity for $k \leq n$. There is an element, which we will denote by $\phi_{n}$, in $T L_{n}$ called the $n$th Jones-Wenzl projector, which is uniquely defined by the following properties. For the original reference where the projector was defined and studied, see [Wen87]. Whenever $n$ is specified we will simply refer to this element as the Jones-Wenzl projector.

(i) $\grave{中}_{n} \cdot e_{n}^{i}=e_{n}^{i} \cdot \dot{中}_{n}=0$ for $1 \leq i \leq n-1$.

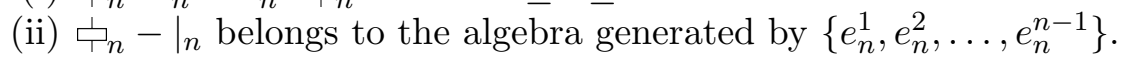

(iii) 中 $_{n} \cdot$ 中 $_{n}=$ 中 $_{n}$. 
(iv) The image of 中 $_{n}$ in $\mathcal{S}\left(\mathbb{R}^{2}\right)$, obtained by embedding the disc $\mathcal{D}^{2}$ in the plane and then joining the $n$ boundary points on the top with those on the bottom with $n$ disjoint planar parallel arcs outside of $\mathcal{D}^{2}$, is equal to

$$
\frac{(-1)^{n}\left(A^{2(n+1)}-A^{-2(n+1)}\right)}{A^{2}-A^{-2}} \cdot \text { the empty diagram in } \mathbb{R}^{2} .
$$

To simplify notation, we will let

$$
\triangle_{n}=\frac{(-1)^{n}\left(A^{2(n+1)}-A^{-2(n+1)}\right)}{A^{2}-A^{-2}} .
$$

From the defining properties, the Jones-Wenzl projector also satisfies a recursion relation (1) and other identities (2) and (3) as indicated in the following figures.
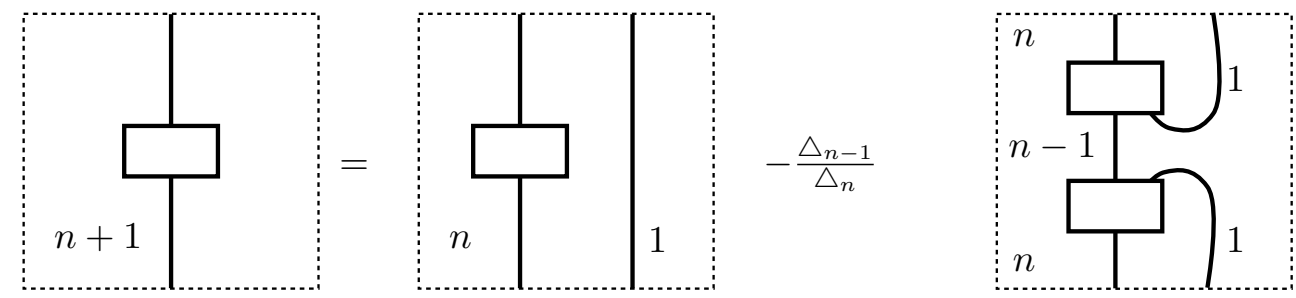

FiguRE 2. A recursive relation for the Jones-Wenzl projector.
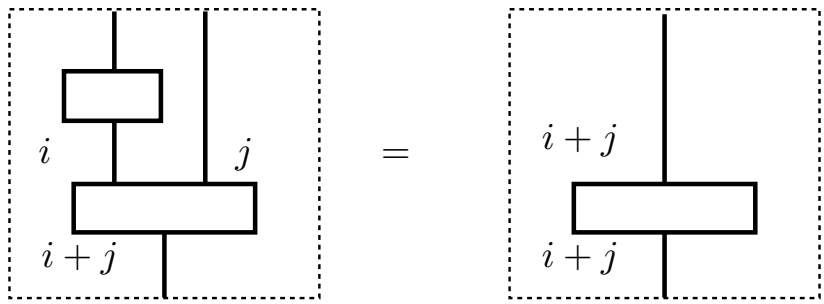

Figure 3. An identity for the Jones-Wenzl projector.

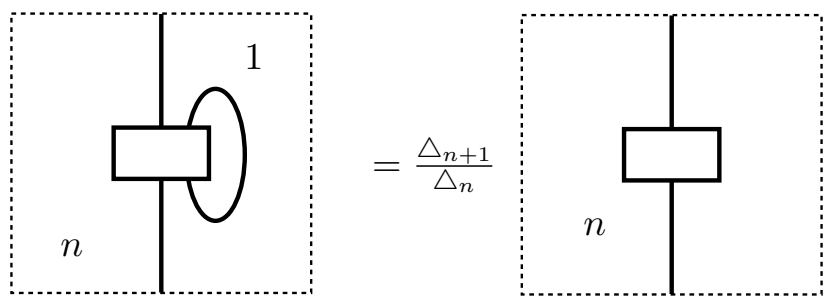

Figure 4. Another identity for the Jones-Wenzl projector.

Definition 2.2. Let $D$ be a diagram of a link $K \subset S^{3}$ with $k$ components. For each component $D_{i}$ for $i \in\{1, \ldots, k\}$ take an annulus $A_{i}$ via the blackboard framing. Let $\mathcal{S}\left(S^{1} \times I\right)$ be the linear skein of the annulus with no points marked on its boundary. Let

$$
f_{D}: \underbrace{\mathcal{S}\left(S^{1} \times I\right) \times \cdots \times \mathcal{S}\left(S^{1} \times I\right)}_{k \text { times }} \rightarrow \mathcal{S}\left(\mathbb{R}^{2}\right)
$$


be the map which sends a $k$-tuple of elements $\left(s_{1}, \ldots, s_{k}\right)$ to $S\left(\mathbb{R}^{2}\right)$ by immersing in the plane the collection of annuli containing the skeins such that the over- and under-crossings of $D$ are the overand under-crossings of the annuli. For $n \geq 2$, the $n$th unreduced colored Jones polynomial $J_{K}^{n}(q)$ may be defined as

$$
J_{K}^{n}(q):=\left.\left((-1)^{n-1} q^{\frac{(n-1)^{2}+2(n-1)}{4}}\right)^{\omega(D)}\langle f_{D} \underbrace{\left(\bigcap_{n-1}^{\bigcap_{n-1}, \cdots, \bigcap_{n-1}^{\bigcap_{n}}}\right)}_{k \text { times }}\rangle\right|_{q^{1 / 4}=A^{-1}},
$$

where $\langle D\rangle$ for a linear skein in $\mathcal{S}\left(\mathbb{R}^{2}\right)$ is the polynomial in $A$ multiplying the empty diagram after resolving crossings and removing disjoint circles of $D$ using the skein relations of Definition 2.1. Note that this gives $J_{\bigcirc}^{n}(q)=\left.\triangle_{n-1}\right|_{q^{1 / 4}=A^{-1}}$ as the normalization for the value of the colored Jones polynomial of the unknot. To simplify notation, we will write

$$
D_{\text {吊 }}^{n-1}=f_{D}\left(\bigcap_{n-1} \bigcap_{n-1}, \cdots, \bigcap_{n-1}\right)
$$

for the rest of this paper.

2.2. Semi-adequate links. Let $D$ be a diagram of a link $K$ in $S^{3}$. A Kauffman state is a choice of replacing every crossing of $D$ by the +- or --resolution as in Figure 5 , with the dashed segment recording the location of the crossing before the replacement.

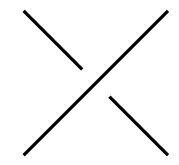

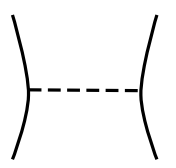

+-resolution

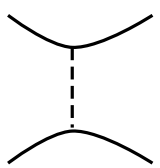

--resolution

FiguRE 5. +- and --resolutions of a crossing.

Applying a Kauffman state results in a set of disjoint circles called state circles. We form a $\sigma$-state graph $s_{\sigma}(D)$ for each Kauffman state $\sigma$ by letting the resulting state circles be vertices and the segments be edges. The all-+ state graph $s_{+}(D)$ comes from the Kauffman state which chooses the +-resolution at every crossing of $D$. Similarly, the all-- state graph $s_{-}(D)$ comes from the Kauffman state which chooses the --resolution at every crossing of $D$.

Definition 2.3. A link diagram $D$ is +-adequate if its all-+ state graph has no one-edged loops (a one-edged loop is an edge that has both ends on the same vertex). A diagram is --adequate if its mirror image is +-adequate.

Definition 2.4. A link $K$ is semi-adequate (+-or--adequate) if it admits a diagram that is + or --adequate.

We consider the following combinatorial data of an oriented link diagram $D$ and a Kauffman state $\sigma$ on $D$.

- $c(D):=$ The number of crossings in $D$.

- $\omega(D):=$ The writhe of $D$.

- $\left|s_{\sigma}(D)\right|:=$ The number of vertices in the $\sigma$-state graph of $D$.

- $\operatorname{sgn}(\sigma):=\operatorname{sgn}_{+}(\sigma)-\operatorname{sgn}_{-}(\sigma)$, where

$\operatorname{sgn}_{+}(\sigma):=$ The number of crossings where the +-resolution is chosen in $\sigma$, and $\operatorname{sgn}_{-}(\sigma):=$ The number of crossings where the --resolution is chosen in $\sigma$. 
We will use the special notations "sgn(+)" and "sgn(-)" for the sign sgn of the all-+ state, and the sign of the all-- state, respectively. Let

$$
H_{n}(D)=n^{2} c(D)+2 n\left|s_{+}(D)\right|
$$

and

$$
h_{n}(D)=-\frac{1}{4} H_{n-1}(D)+\frac{(n-1)^{2}+2(n-1)}{4} \omega(D) .
$$

We use the following lemmas from skein theory involving the notion of adequacy. The degree of a rational function that we consider is the maximum power of the Laurent series expression of the function with the power of each term in the series bounded from above.

Definition 2.5. Let $\mathcal{S}$ be a skein in $\mathcal{S}\left(\mathbb{R}^{2}\right)$ with crossings. Let $\sigma$ and $\sigma^{\prime}$ be Kauffman states on the crossings of $\mathcal{S}$. We say that there is a sequence of states from $\sigma$ to $\sigma^{\prime}$ if there is a finite sequence $\sigma_{1}=\sigma, \sigma_{2}, \ldots, \sigma_{f}=\sigma^{\prime}$ such that for each $i$, there is a distinct crossing $x_{i}$ on which $\sigma_{i}$ chooses the $A$-resolution and $\sigma_{i+1}$ chooses the $B$-resolution, and they are otherwise the same on every other crossing of $\mathcal{S}$.

Note that there is a sequence of states from the all-+ state to any other state.

Definition 2.6. Let $\mathcal{S}$ be a skein in $\mathcal{S}\left(\mathbb{R}^{2}\right)$ which may have crossings and may be decorated by Jones-Wenzl projectors, we will denote by $\overline{\mathcal{S}}$ the skein obtained from $\mathcal{S}$ where every projector is replaced by the identity.

Lemma 2.7. [Lic97, Lemma 5.6] Let $\sigma$ and $\sigma^{\prime}$ be two Kauffman states on a skein $\mathcal{S} \in \mathcal{S}\left(\mathbb{R}^{2}\right)$ with a sequence of states $\left\{\sigma_{i}\right\}_{i=1}^{f}$ from $\sigma$ to $\sigma^{\prime}$, and let $\overline{\mathcal{S}_{\sigma}}$ and $\overline{\mathcal{S}_{\sigma^{\prime}}}$ be the skeins resulting from applying the Kauffman states $\sigma$ and $\sigma^{\prime}$, respectively, to $\mathcal{S}$ and replacing every Jones-Wenzl projector by the identity. Suppose $m \geq 0$ is the number of pairs $\left(\sigma_{i}, \sigma_{i+1}\right)$ in the sequence where the number of circles in $\overline{\mathcal{S}_{\sigma_{i+1}}}$ is one fewer than that of $\overline{\mathcal{S}_{\sigma_{i}}}$, then

$$
\operatorname{deg}\left(A^{\operatorname{sgn}\left(\sigma^{\prime}\right)}\left\langle\overline{\mathcal{S}_{\sigma^{\prime}}}\right\rangle\right) \leq \operatorname{deg}\left(A^{\operatorname{sgn}(\sigma)}\left\langle\overline{\mathcal{S}_{\sigma}}\right\rangle\right)-4 m .
$$

Note that from $\sigma_{i}$ to $\sigma_{i+1}$ in a sequence, either a pair of circles is merged or a circle is split into two.

The definition of an adequate skein is due to Armond [Arm13.

Definition 2.8. Let $\mathcal{S} \in \mathcal{S}\left(\mathbb{R}^{2}\right)$ be a crossing-less skein decorated by Jones-Wenzl projectors 中 $_{n}$. Consider the skein $\overline{\mathcal{S}}$ constructed from $\mathcal{S}$ by replacing each of the Jones-Wenzl projectors by the identity in $T L_{n}$. Consider the regions in $\overline{\mathcal{S}}$ where the projectors had previously been. We say that $\mathcal{S}$ is adequate if no circle in $\overline{\mathcal{S}}$ passes through any of these regions more than once.

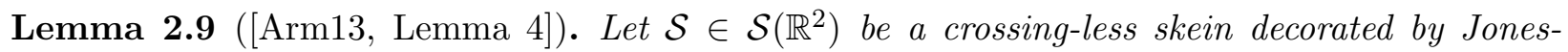
Wenzl projectors $\dot{\mathrm{C}}_{n}$, and let $\overline{\mathcal{S}}$ be the skein obtained by replacing each Jones-Wenzl projector by the identity element $\left.\right|_{n}$, then

$$
\operatorname{deg}\langle\mathcal{S}\rangle \leq \operatorname{deg}\langle\overline{\mathcal{S}}\rangle
$$

and equality is achieved when $\mathcal{S}$ is adequate.

The next lemma follows immediately from [Lic97, Lemma 5.6].

Lemma 2.10. Let $\mathcal{S} \in \mathcal{S}\left(\mathbb{R}^{2}\right)$ be a skein with crossings decorated by the Jones-Wenzl projector 中 $_{n}$ for some fixed $n$, and let $\mathcal{S}_{+}$be the skein resulting from applying the all-+ Kauffman state on the crossings of $\mathcal{S}$, then

$$
\operatorname{deg}\langle\mathcal{S}\rangle \leq \operatorname{deg}\left(A^{\operatorname{sgn}(+)}\left\langle\overline{\mathcal{S}_{+}}\right\rangle\right)
$$


Proof. Let $\mathcal{S}_{\sigma}$ be the skein resulting from applying the Kauffman state $\sigma$ on the crossings of $\mathcal{S}$. Then

$$
\langle\mathcal{S}\rangle=\sum_{\sigma} A^{\operatorname{sgn}(\sigma)}\left\langle\mathcal{S}_{\sigma}\right\rangle
$$

By Lemma 2.9,

$$
\operatorname{deg}\left\langle\mathcal{S}_{\sigma}\right\rangle \leq \operatorname{deg}\left\langle\overline{\mathcal{S}_{\sigma}}\right\rangle
$$

Now

$$
\operatorname{deg}\left(A^{\operatorname{sgn}(\sigma)}\left\langle\overline{\mathcal{S}_{\sigma}}\right\rangle\right) \leq \operatorname{deg}\left(A^{\operatorname{sgn}(+)}\left\langle\overline{\mathcal{S}_{+}}\right\rangle\right)
$$

by considering the link diagram $\overline{\mathcal{S}}$ and applying Lemma 2.7 , since there is a sequence of states from the all- + state to any other state.

We use Lemma 2.9 and 2.10 to give a proof of the following fact used to establish the degree of the colored Jones polynomial of a +-adequate link [Lic97].

Corollary 2.11. Let $D$ be a link diagram and $D_{\perp}^{n}$ be the $n$-blackboard cable of $D$ with each component decorated by a Jones-Wenzl projector as in Definition 2.2, then

$$
\operatorname{deg}\left\langle D_{\text {吊 }}^{n}\right\rangle \leq H_{n}(D) \text {. }
$$

Therefore,

$$
h_{n}(D) \leq d(n)
$$

and equality is achieved when $D$ is +-adequate.

Proof. Let $\sigma$ be a Kauffman state on the set of crossings of $D_{\perp}^{n}$, with $S_{\sigma}^{n}$ the skein resulting from applying the state $\sigma$ and $S_{+}^{n}$ the skein resulting from applying the all-+ state. The inequality follows from writing

$$
\left\langle D_{\perp_{p}}^{n}\right\rangle=\sum_{\sigma} A^{\operatorname{sgn}(\sigma)}\left\langle\mathcal{S}_{\sigma}^{n}\right\rangle,
$$

Lemma 2.10, and recognizing that $\operatorname{deg}\left(A^{\operatorname{sgn}(+)}\left\langle\overline{S_{+}^{n}}\right\rangle\right)=H_{n}(D)$. The skein $\mathcal{S}_{+}^{n}$ is adequate, so by Lemma 2.9 we have

$$
\operatorname{deg}\left\langle S_{+}^{n}\right\rangle=\operatorname{deg}\left\langle\overline{S_{+}^{n}}\right\rangle .
$$

Now if $D$ is +-adequate, then $D^{n}$ is also +-adequate, which one can directly check as in Lic97, Lemma 5.12]. A sequence of states from the all-+ state to any other state necessarily contains a pair which merges a pair of circles. This implies

$$
\operatorname{deg}\left(A^{\operatorname{sgn}(\sigma)}\left\langle\overline{\mathcal{S}_{\sigma}^{n}}\right\rangle\right)<\operatorname{deg}\left(A^{\operatorname{sgn}(+)}\left\langle\overline{\mathcal{S}_{+}^{n}}\right\rangle\right) .
$$

Thus

$$
\operatorname{deg}\left\langle D_{\Gamma_{p}}^{n}\right\rangle=\operatorname{deg}\left(A^{\operatorname{sgn}(+)}\left\langle\overline{\mathcal{S}_{+}^{n}}\right\rangle\right)=H_{n}(D)
$$

\section{Proof of Theorem 1.1}

Throughout this section, we assume that $D$ is not +-adequate, and therefore, it has a one-edged loop in its all-+ state graph. Recall that, as in Definition 2.2 , we may obtain $J_{K}^{n}(q)$ by evaluating the Kauffman bracket on $D_{\text {中, }}^{n}$, the $n$-blackboard cable $D^{n}$ decorated by a Jones-Wenzl projector. In our case, we will consider the decoration by four Jones-Wenzl projectors around a fixed cabled crossing $c$, which corresponds to a loop in the all-+ state graph of $D$. See Figure 6 for what is meant by cabling a crossing and Figure 7 for an example of a chosen crossing framed by projectors. Without loss of generality, we assume the loop is attached on the inside of a state circle $S$ of $s_{+}(D)$. Since this skein is obtained from $D_{\dot{\phi}}^{n}$ by doubling and sliding the projector on each component using 
the defining properties of the projector, it is equivalent to $D_{\text {p }}^{n}$ and we will also denote the resulting skein by $D_{\perp}^{n}$ by a slight abuse of notation.

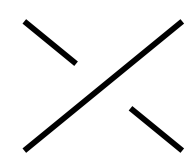

cabling

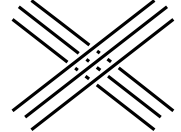

Figure 6. A crossing and its 3-cable.

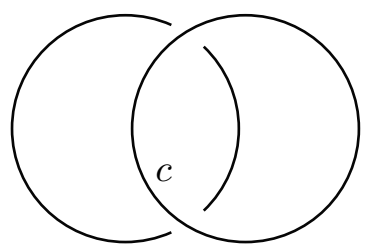

A non +-adequate link diagram

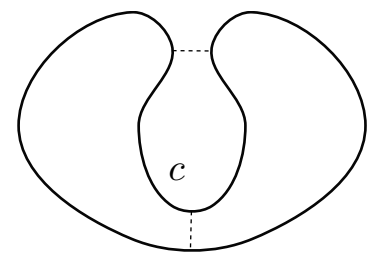

The all-+ state

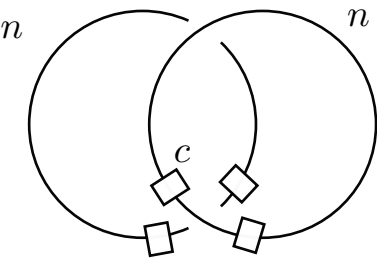

The chosen crossing decorated with projectors

FiguRE 7. We put four Jones-Wenzl projectors around a single crossing $c$.

Theorem 1.1 will follow from the equivalent statement given below for the Kauffman bracket with variable $A$. Recall that by Corollary 2.11, for any link diagram we have

$$
\operatorname{deg}\left\langle D_{\text {吊 }}^{n}\right\rangle \leq H_{n}(D) \text {. }
$$

Theorem 3.1. Let $K$ be a link with diagram $D$ and $H_{n}(D)$ be defined from $D$ as in (5). If $D$ is not + -adequate, then $\operatorname{deg}\left\langle D_{\perp}^{n}\right\rangle \leq H_{n}(D)-4(n-1)$ for $n \geq 1$.

Henceforth, we will work exclusively with the Kauffman bracket.

Let $\mathcal{S}$ be a skein with crossings in $\mathcal{S}\left(\mathbb{R}^{2}\right)$ which may or may not be decorated by Jones-Wenzl projectors. We denote by $\mathcal{S}_{\sigma}$ the crossing-less skein obtained by applying a Kauffman state $\sigma$ to the crossings of $\mathcal{S}$. A $\sigma$-state graph $s_{\sigma}(\mathcal{S})$ is then the set of disjoint circles with segments as before, except for the presence of projectors.

Let $D$ be a link diagram and consider a skein $\mathcal{S}_{\sigma}^{n}$ obtained by applying a Kauffman state $\sigma$ to the crossings of $D_{\square}^{n}$. We have the following state sum for the Kauffman bracket of $D_{\text {म }}^{n}$.

$$
\left\langle D_{\text {मे }}^{n}\right\rangle=\sum_{\sigma} A^{\operatorname{sgn}(\sigma)}\left\langle\mathcal{S}_{\sigma}^{n}\right\rangle .
$$

Strategy for proof of Theorem 3.1. The strategy for the proof of Theorem 3.1 is to characterize the states in the above equation relevant to the last $n-2$ coefficients of $\left\langle D_{\text {中 }}^{n}\right\rangle$ from $H_{n}(D)$ for $D$ a non +-adequate diagram. Then, Lemma 3.3 and Lemma 3.6 are used to relate these states to the skein of a diagram of the unknot in Definition 3.4. which establishes that these last $n-2$ coefficients are trivial by Lemma 3.5 .

We make the following definition which generalizes the $\doteq_{n}$ equivalence in [Arm13, Pg. 1].

Definition 3.2. Let $s$ and $m$ be two integers $\geq 0$ and $P_{1}(A)$ and $P_{2}(A)$ be two Laurent series in A. We write

$$
P_{1}(A) \stackrel{\doteq^{s}}{m} P_{2}(A)
$$

if and only if the coefficients of $A^{s}, A^{s-4(1)}, \ldots, A^{s-4(m-1)}$ and $A$ 's with power $>s$ in $P_{1}(A)$ agree with those of $P_{2}(A)$. For example, $2 A^{9}-A^{5}+A^{1} \doteq_{2}^{9} 2 A^{9}-A^{5}+4 A^{1}$. For two skeins $\mathcal{S}_{1}, \mathcal{S}_{2} \in \mathcal{S}\left(\mathbb{R}^{2}\right)$, we write $\mathcal{S}_{1} \doteq_{m}^{s} \mathcal{S}_{2}$ if $\left\langle\mathcal{S}_{1}\right\rangle \doteq_{m}^{s}\left\langle\mathcal{S}_{2}\right\rangle$. 
The following lemma is an important variant of [Arm13, Lemma 10].

Lemma 3.3. Let $0 \leq k \leq n$.

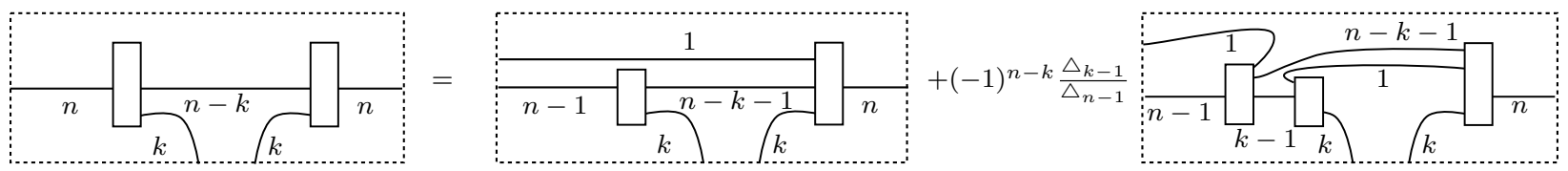

Proof. Similar to the proof of [Arm13, Lemma 10], we apply the recursive relation (1) to the left projector in the picture on the left side of the equality above to get the following equality as indicated in (8).

(8)

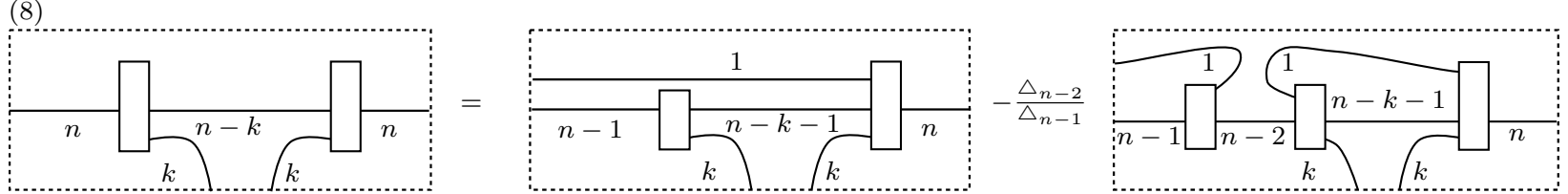

For $n-k>1$, we apply the recursive relation again to the middle projector in the last picture in the above equation. It is clear that the second term in the resulting sum is zero, due to property

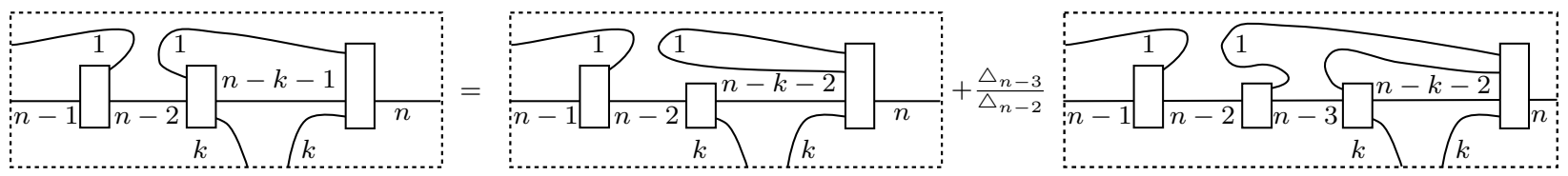

(i) of the Jones-Wenzl projector. Sliding to the left the second projector from the left in the last picture above by (2), and combining with (8), we see that we get the lemma by repeated expansion $((n-k)$ times $)$ via the recursion relation.

We consider a particular type of skeins from a diagram of the unknot and study their $\doteq_{m}^{s}$ equivalences using Lemma 3.3 .

Definition 3.4. Let $U_{\text {p }}^{n}$ be the $n$-blackboard cable of the diagram of the unknot with one left-hand

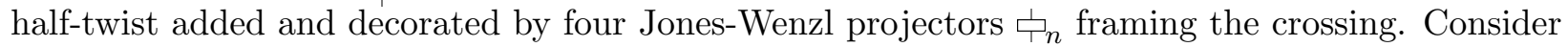
the set of disjoint circles $\overline{U_{+}^{n}}$ from applying the all-+ Kauffman state to the crossings of $U_{\text {中 }}^{n}$, and then replacing each Jones-Wenzl projector by the identity. Label each of them, innermost first, as $S_{1}, \ldots, S_{n}$. For $1 \leq j<n$, let $U^{n, j}$ be the skein obtained from $U_{+}^{n}$ by removing all circles and segments resulting from applying the all-+ Kauffman state outside of $S_{j}$, and replacing each remaining segment by the corresponding crossing before choosing the +-resolution. See Figure 8 for an example. 

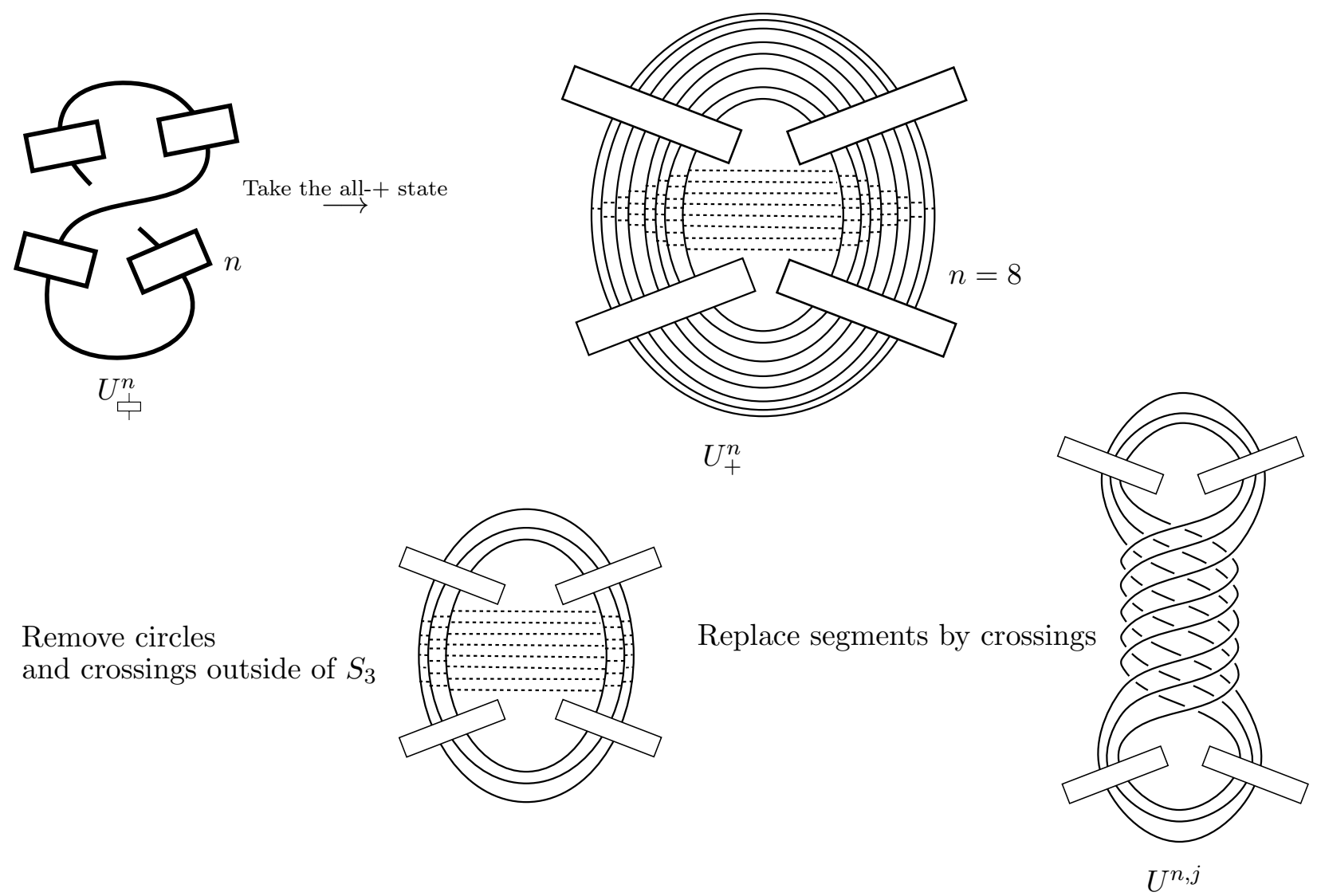

Figure 8. An example of $U^{n, j}$ where $j=3$ and $n=8=2 j+2$.

The key lemma below computes a degree bound on $\left\langle U^{n, j}\right\rangle$ for $U^{n, j}$ from Definition 3.4 .

Lemma 3.5. Let $U_{+}^{n, j}$ be the skein obtained from $U^{n, j}$ as defined in Definition 3.4 by applying the all-+ Kauffman state to its crossings, then we have for $1 \leq j<n$,

$$
\operatorname{deg}\left\langle U^{n, j}\right\rangle \leq n^{2}-(n-j)(n-j+1)+\operatorname{deg}\left\langle\overline{U_{+}^{n, j}}\right\rangle-4 j
$$

Proof. Firstly, the number of crossings of $U^{n, j}$ is $n^{2}-(n-j)(n-j+1)$, thus

$$
\operatorname{deg}\left\langle U^{n, j}\right\rangle \leq \underbrace{\left.n^{2}-(n-j)(n-j+1)+\operatorname{deg} \overline{\left\langle U_{+}^{n, j}\right.}\right\rangle}_{\text {general upper bound }}
$$

by Lemma 2.10. Write $n=n^{\prime} j+r$ with the smallest nonnegative remainder $r$. We have $n^{\prime} \geq 1$ because of the assumption that $j<n$. The skein $U^{n, j}$ has $n^{\prime}$ full kinks on $j$ strands from examining the braid word and removing crossings with the presence of the projectors. Removing each full twist on $j$ strands will decrease the degree by $j^{2}+2 j$ [Lic97, Lemma 14.1] in addition to removing $j^{2}$ crossings. Thus the degree decreases from the upper bound $\left.n^{2}-(n-j)(n-j+1)+\operatorname{deg} \overline{\left\langle U_{+}^{n, j}\right.}\right\rangle$ from taking the all-+ state by $n^{\prime}\left(2 j^{2}+2 j\right) \geq 4 j$ for $n^{\prime}, j \geq 1$.

Now we consider skeins which can be reduced to $U^{n, j}$ through $\doteq_{*}^{s}$-equivalences.

Lemma 3.6. Suppose that we have a skein $\mathcal{S}$ in $\mathcal{S}\left(\mathbb{R}^{2}\right)$ of the form shown below in Figure 9 . Consider the set of circles of $\overline{\mathcal{S}}$ and number the circles, innermost first, as $S_{1}, \ldots, S_{j}$, where $j=$ 
$n-\left(\ell+\ell^{\prime}\right)$. Let $L^{n, j}$ be the subset of the crossings of $U^{n, j}$ inside $S_{j}$, and let $\left|L^{n, j}\right|$ be the number of crossings in $L^{n, j}$.

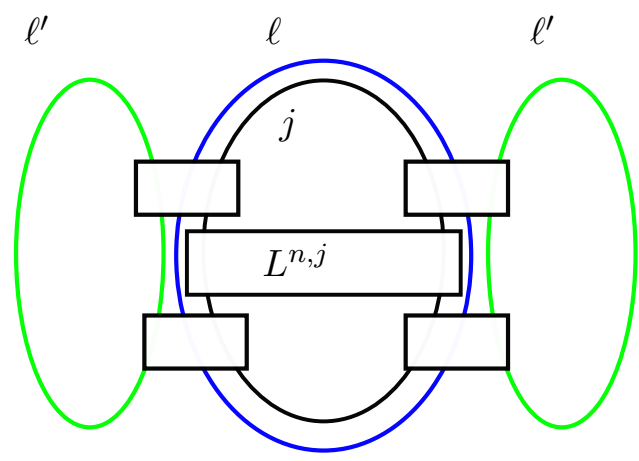

Figure 9. The skein $\mathcal{S}$ composed of crossings from $U^{n, j}$ and $\ell+2 \ell^{\prime}$ circles attached to the projectors.

Then,
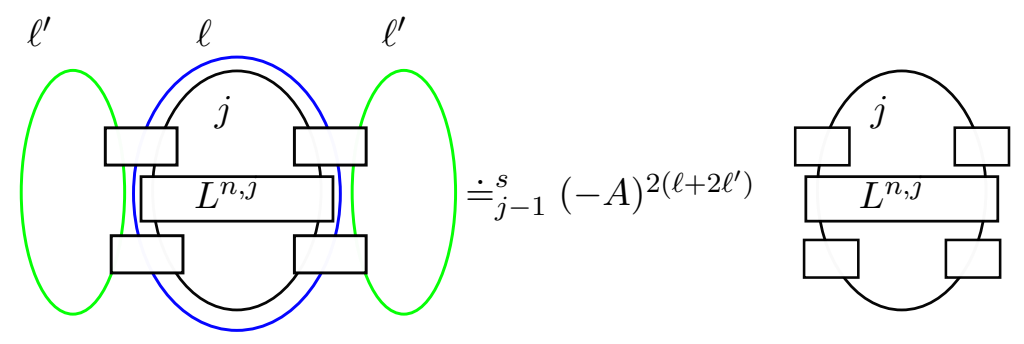

where $s=\left|L^{n, j}\right|+\operatorname{deg}\left\langle\overline{\mathcal{S}_{+}}\right\rangle$.

Proof. We apply Lemma 3.3 to the pair of projectors on the right side of $\mathcal{S}$.

$(11)$

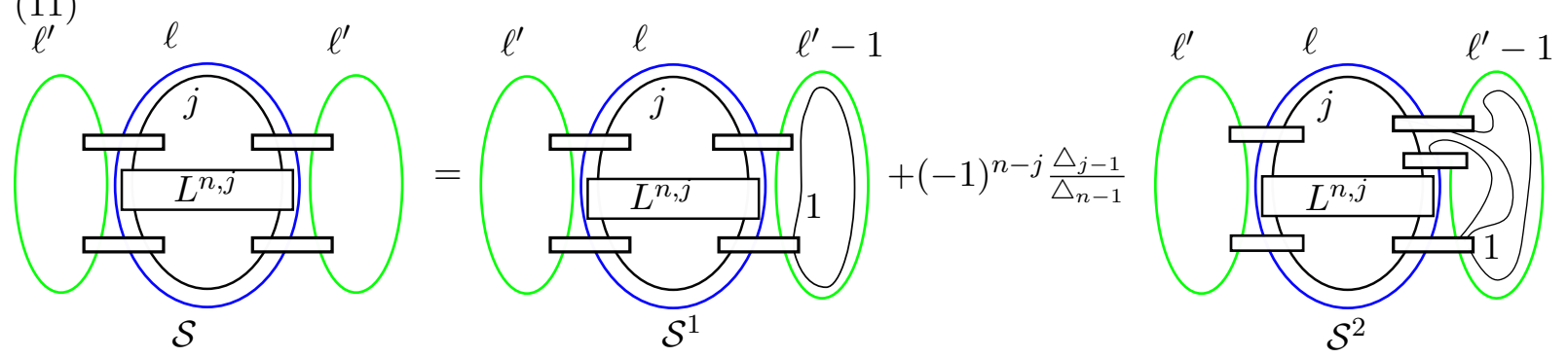

We write $\mathcal{S}^{1}$ for the first term of the sum. For the second term $\mathcal{S}^{2}$ of the sum, we have the local picture shown below where Lemma 3.3 was applied. 


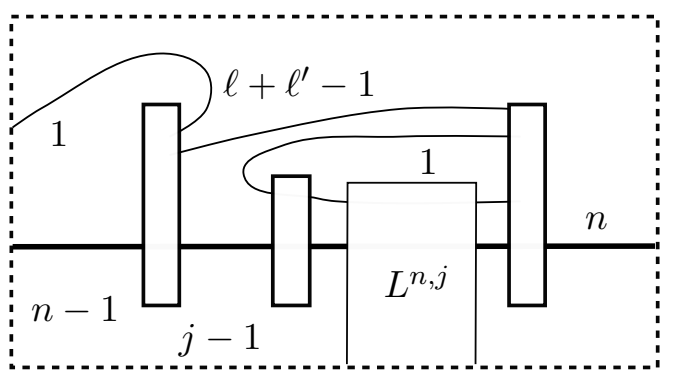

We compare the maximum degree of the Kauffman bracket of $\mathcal{S}^{1}$ and $\mathcal{S}^{2}$. Note first that

$$
\begin{gathered}
\operatorname{deg}\left(A^{\operatorname{sgn}(+)}\left\langle\overline{\mathcal{S}_{+}^{1}}\right\rangle\right)=\operatorname{deg}\left(A^{\operatorname{sgn}(+)}\left\langle\overline{\mathcal{S}_{+}}\right\rangle\right)=s, \text { where } \operatorname{sgn}(+)=\left|L^{n, j}\right| . \text { Now } \\
\operatorname{deg} \frac{\triangle_{j-1}}{\triangle_{n-1}}=\operatorname{deg} \frac{\triangle_{n-\ell-\ell^{\prime}-1}}{\triangle_{n-1}}=-2\left(\ell+\ell^{\prime}\right) .
\end{gathered}
$$

Let $\sigma$ be a Kauffman state on the crossings of $\mathcal{S}^{2}$, then

$$
\left\langle\mathcal{S}^{2}\right\rangle=\sum_{\sigma} A^{\operatorname{sgn}(\sigma)}\left\langle\mathcal{S}_{\sigma}^{2}\right\rangle
$$

In order to estimate the maximum degree of $\left\langle\mathcal{S}^{2}\right\rangle$ relative to $A^{\operatorname{sgn}(+)}\left\langle\overline{\mathcal{S}_{+}^{1}}\right\rangle$, we estimate the degree of each term in the sum above.

Let $k$ be the largest number $\in\{1, \ldots, j\}$ such that $\sigma$ chooses the --resolution on a crossing of $L^{n, j}$ between $S_{k-1}$ and $S_{k}$. The state $\sigma$ has to choose the --resolution for some crossing between $S_{i-1}$ and $S_{i}$ for all $i \in\{2, \ldots, k\}$. Otherwise, there would be a cap or a cup composed with a projector that would make $\left\langle\mathcal{S}_{\sigma}^{2^{\prime}}\right\rangle=0$. In the pictorial calculations to follow, we will denote by $L_{\sigma}$ the result of applying $\sigma$ to the crossings in $L^{n, j}$. Applying Lemma 3.3 , we have

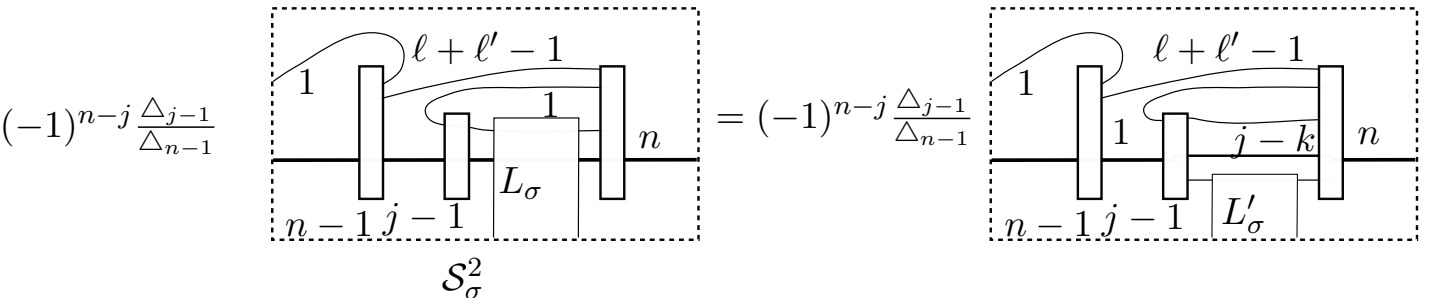

$$
\begin{aligned}
& =\left.(-1)^{n-k} \frac{\triangle_{k-1}}{\triangle_{n-1}} \prod_{n-1 k-1}^{1}\right|_{\bigsqcup_{L_{\sigma}^{\prime}}^{\prime}} ^{n-k-1} n
\end{aligned}
$$

where $L_{\sigma}^{\prime}$ is the result of applying $\sigma$ to the set of crossings of $L^{n, j}$ inside of $S_{k}$. Now we estimate $\operatorname{sgn}(\sigma)+2\left|\overline{\mathcal{S}_{\sigma}^{2^{\prime}}}\right|$ relative to $\operatorname{sgn}(+)+2\left|\overline{\mathcal{S}_{+}^{2^{\prime}}}\right|$. The condition that $\sigma$ has to choose the - -resolution for a crossing between $S_{i-1}$ and $S_{i}$ for all $i \in\{2, \ldots, k\}$ forces $\operatorname{deg}\left(A^{\operatorname{sgn}(\sigma)}\left\langle\overline{\mathcal{S}_{\sigma}^{2^{\prime}}}\right\rangle\right) \leq \operatorname{deg}\left(A^{\operatorname{sgn}(+)}\left\langle\overline{\mathcal{S}_{+}^{2^{\prime}}}\right\rangle\right)-$ $4(k-1)$ by Lemma 2.7. This is because each change of resolution from + - to -- merges $S_{i}$ and $S_{i+1}$. Now the fact that $\left|\overline{\mathcal{S}_{+}^{2^{\prime}}}\right|=\left|\overline{\mathcal{S}_{+}^{1}}\right|-(n-k)$ and $\operatorname{deg} \frac{\triangle_{k-1}}{\triangle_{n-1}}=-2(n-k)$ then gives

$$
\operatorname{deg}\left(A^{\operatorname{sgn}(\sigma)}\left\langle\overline{\mathcal{S}_{\sigma}^{2 \prime}}\right\rangle\right) \leq \operatorname{deg}\left(A^{\operatorname{sgn}(+)}\left\langle\overline{\mathcal{S}_{+}^{1}}\right\rangle\right)-4(n-1) .
$$

Thus

$$
\mathcal{S} \doteq{ }_{n-1}^{s} \mathcal{S}^{1}
$$


At this point either we are dealing with a circle in the $\ell$ circles and we can apply Lemma 3.3 again to the bottom pair of projectors, or, if we are dealing with a circle in a pair of $\ell^{\prime}$ pairs of circles, we simply have a loop attached to a Jones-Wenzl projector that we can pull off via an $\dot{=}_{n-1}^{s}$ equivalence using (3) by rewriting

$$
\begin{aligned}
\frac{\triangle_{n+1}}{\triangle_{n}} & =-\frac{A^{2(n+2)}-A^{-2(n+2)}}{A^{2(n+1)}-A^{-2(n+1)}}=-A^{2} \frac{1-A^{-4(n+2)}}{1-A^{-4(n+1)}} \\
& =-A^{2}\left(1+A^{-4(n+1)}+\cdots+\text { terms with powers lower than }-4 n\right) .
\end{aligned}
$$

See Figure 11 for an illustration of both of these cases.

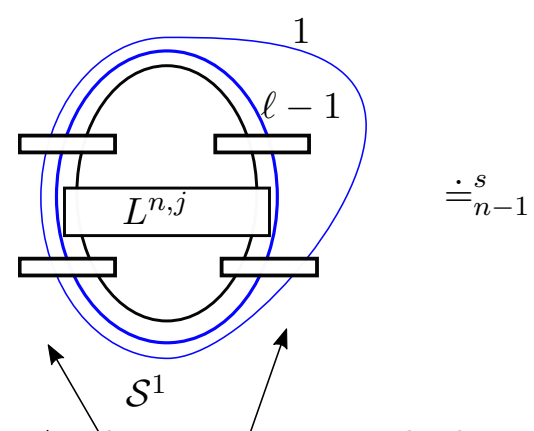

Apply Lemma 3.3 to the bottom pair of projectors
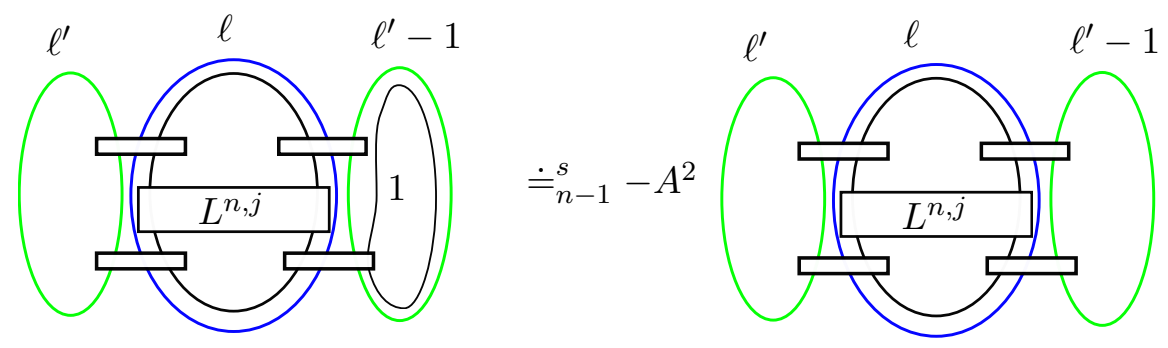

FiguRE 11. The case when $\ell>0$.

We repeat this process to detach each of the $2 \ell^{\prime}+\ell$ circles, keeping track of the change in the $\doteq$-equivalences when the number of strands through each projector decreases. This finishes the proof of the lemma.

We now prove Theorem 3.1. Recall that

$$
\left\langle D_{\perp}^{n}\right\rangle=\sum_{\sigma} A^{\operatorname{sgn}(\sigma)}\left\langle\mathcal{S}_{\sigma}^{n}\right\rangle .
$$

Since $H_{n}(D)=\operatorname{deg}\left(A^{\operatorname{sgn}(+)}\left\langle\overline{\mathcal{S}_{+}^{n}}\right\rangle\right)$, it suffices to show that

$$
\operatorname{deg}\left(\sum_{\sigma} A^{\operatorname{sgn}(\sigma)}\left\langle\mathcal{S}_{\sigma}^{n}\right\rangle\right) \leq \operatorname{deg}\left(A^{\operatorname{sgn}(+)}\left\langle\overline{\mathcal{S}_{+}^{n}}\right\rangle\right)-4(n-1) .
$$

This follows from the technical lemma below. 
Lemma 3.7. Let $D$ be a non +-adequate diagram with a crossing c corresponding to a loop inside a state circle in its all-+ state graph. Let $\mathcal{S}_{\sigma}^{n}$ be a skein obtained by applying a Kauffman state $\sigma$ to the crossings of $D_{中}^{n}$, which is decorated with four Jones-Wenzl projectors framing c. Consider $\sigma$ such that

$$
\operatorname{deg}\left(A^{\operatorname{sgn}(\sigma)}\left\langle\overline{\mathcal{S}_{\sigma}^{n}}\right\rangle\right)>\operatorname{deg}\left(A^{\operatorname{sgn}(+)}\left\langle\overline{\mathcal{S}_{+}^{n}}\right\rangle\right)-4(n-1)
$$

We have

$$
\operatorname{deg}\left(\sum_{\sigma \text { satisfying }} A^{\operatorname{sgn}(\sigma)}\left\langle\mathcal{S}_{\sigma}^{n}\right\rangle\right) \leq \operatorname{deg}\left(A^{\operatorname{sgn}(+)}\left\langle\overline{\mathcal{S}_{+}^{n}}\right\rangle\right)-4(n-1) .
$$

By disregarding the skeins from states whose Kauffman brackets have maximum degrees which are too low, we have

$$
\left\langle D_{\bigsqcup^{\prime}}^{n}\right\rangle=\sum_{\sigma} A^{\operatorname{sgn}(\sigma)}\left\langle S_{\sigma}^{n}\right\rangle \dot{=}_{n-1}^{s}\left(\sum_{\sigma \text { satisfying }[13]} A^{\operatorname{sgn}(\sigma)}\left\langle\mathcal{S}_{\sigma}^{n}\right\rangle\right) .
$$

Applying Lemma 3.7 then completes the proof of Theorem 3.1 .

Proof. (of Lemma 3.7)

A Kauffman state $\sigma$ satisfying (13) chooses the +-resolution on all crossings whose corresponding segments lie between $S_{i}, S_{i+1}$ in $\overline{\mathcal{S}_{+}^{n}}$ for some $1 \leq i \leq n-1$. To see this, we compare $\sigma$ to the all-+ state. If $\sigma$ chooses the --resolution at a crossing whose corresponding segment lies between $S_{i}$ and $S_{i+1}$ for every $1 \leq i \leq n-1$, then

$$
\operatorname{deg}\left(A^{\operatorname{sgn}(\sigma)}\left\langle\overline{\mathcal{S}_{\sigma}^{n}}\right\rangle\right) \leq \operatorname{deg}\left(A^{\operatorname{sgn}(+)}\left\langle\overline{\mathcal{S}_{+}^{n}}\right\rangle\right)-4(n-1)
$$

by taking a sequence of states from the all- + state to $\sigma$ where there are $n-1$ pairs of terms in the sequence, each of which merges a pair of circles, and applying Lemma 2.7.

For a Kauffman state $\sigma$ on $D_{\text {中 }}^{n}$, let $j_{\sigma}$ be the largest integer in $\{1, \ldots, n-1\}$ where $\sigma$ chooses the +-resolution for all the crossings corresponding to segments between $S_{i}$ and $S_{i+1}$. In $D_{\perp}^{n}$, the loop crossing $c$ cables to $c^{n}$ crossings. Let $L_{j}$ be the subset of crossings of $c^{n}$ whose corresponding segments in $\mathcal{S}_{+}^{n}$ lie inside $S_{j}$. We define an equivalence relation on the set of skeins with Kauffman brackets satisfying (13): Two skeins $\mathcal{S}_{\sigma}^{n}$ and $\mathcal{S}_{\sigma^{\prime}}^{n}$ resulting from applying the Kauffman states $\sigma$ and $\sigma^{\prime}$ to $D_{\dot{\phi}}^{n}$, respectively, are equivalent, and we write $\mathcal{S}_{\sigma}^{n} \sim \mathcal{S}_{\sigma^{\prime}}^{n}$, if and only if

(a) $j_{\sigma}=j_{\sigma^{\prime}}$

(b) $\sigma$ and $\sigma^{\prime}$ are identical outside of $L_{j_{\sigma}}$.

It is clear that $\sim$ is an equivalence relation. In an equivalence class of $\sigma$, we may decompose $\sigma$ as a disjoint union of Kauffman states $\sigma_{1} \sqcup \sigma_{2}$, where $\sigma_{1}$ is on the crossings in $L_{j_{\sigma}}$, and $\sigma_{2}$ is on the crossings not in $L_{j_{\sigma}}$. So we have $\operatorname{sgn}(\sigma)=\operatorname{sgn}\left(\sigma_{1}\right)+\operatorname{sgn}\left(\sigma_{2}\right)$.

We have

$$
\sum_{\sigma \text { satisfying }} A^{\operatorname{sgn}(\sigma)}\left\langle\mathcal{S}_{\sigma}^{n}\right\rangle=\sum_{C \text { an equivalence class of } \sim} \sum_{\sigma \in C} A^{\operatorname{sgn}(\sigma)}\left\langle\mathcal{S}_{\sigma}^{n}\right\rangle .
$$

Since $\operatorname{sgn}\left(\sigma_{2}\right)$ is identical across $C$, this is equal to

$$
\sum_{\sigma \text { satisfying }} A^{\operatorname{sgn}(\sigma)}\left\langle\mathcal{S}_{\sigma}^{n}\right\rangle=\sum_{C \text { an equivalence class of } \sim} A^{\operatorname{sgn}\left(\sigma_{2}\right)} \sum_{\sigma \in C} A^{\operatorname{sgn}\left(\sigma_{1}\right)}\left\langle\mathcal{S}_{\sigma}^{n}\right\rangle .
$$


Fix an equivalence class $C$ and let $\mathcal{S}^{n, C}$ be the skein resulting from applying $\sigma_{2}$ to the crossings not in $L_{j_{\sigma}}$ for $\sigma \in C$. Then

$$
\sum_{\sigma \text { satisfying } 13 \text { [13 }} A^{\operatorname{sgn}(\sigma)}\left\langle\mathcal{S}_{\sigma}^{n}\right\rangle=\sum_{C \text { an equivalence class of } \sim} A^{\operatorname{sgn}\left(\sigma_{2}\right)}\left\langle\mathcal{S}^{n, C}\right\rangle .
$$

The component $\mathcal{S}$ decorated by projectors in $\mathcal{S}^{n, C}$ may be isotoped to have the form of Figure 9 with $n-\left(\ell+\ell^{\prime}\right)=j_{\sigma}$ for $\sigma \in C$, since otherwise there is a cap or a cup composed with a projector, resulting in a 0 skein. Let $\ell^{\prime}$ be the number of pairs of circles, each going through two projectors and $\ell$ be the number of circles through all four projectors outside of $S_{j_{\sigma}}$. Let $s^{\prime}=\left|L_{j_{\sigma}}\right|+\operatorname{deg}\left\langle\overline{\mathcal{S}_{+}^{n, C}}\right\rangle$, where $\mathcal{S}_{+}^{n, C}$ is the skein resulting from applying the all-+ state to the crossings of $\mathcal{S}^{n, C}$, and let $\left|\sigma_{2}\right|$ be the number of circles disjoint from the component decorated by the projectors in $\mathcal{S}^{n, C}$. Now it follows directly from Lemma 3.6 that

$$
\left\langle\mathcal{S}^{n, C}\right\rangle \doteq_{n-1}^{s^{\prime}}\left(-A^{-2}-A^{2}\right)^{\left|\sigma_{2}\right|}(-A)^{2\left(\ell+2 \ell^{\prime}\right)}\left\langle U^{n, j_{\sigma}}\right\rangle,
$$

where $U^{n, j_{\sigma}}$ is the skein as defined by Definition 3.4 .

By Lemma 3.5 ,

This implies

$$
\operatorname{deg}\left\langle U^{n, j_{\sigma}}\right\rangle \leq\left|L_{j_{\sigma}}\right|+\operatorname{deg}\left\langle\overline{U_{+}^{n, j_{\sigma}}}\right\rangle-4 j_{\sigma}
$$

$$
\operatorname{deg}\left\langle\mathcal{S}^{n, C}\right\rangle \leq s^{\prime}-4 j_{\sigma} .
$$

Take a sequence of states from the all- + state to $\sigma=\sigma_{2} \sqcup \sigma_{1}$. Since $\sigma_{2}$ chooses the --resolution on a crossing between $S_{i}$ and $S_{i+1}$ for every $i \in\left\{j_{\sigma}, \ldots, n-1\right\}$, we have by applying Lemma 2.7 that

$$
s^{\prime}+\operatorname{sgn}\left(\sigma_{2}\right)+2\left|\sigma_{2}\right| \leq \operatorname{sgn}(+)+\operatorname{deg}\left\langle\overline{\mathcal{S}_{+}^{n}}\right\rangle-4\left(n-j_{\sigma}-1\right) .
$$

Taken with 190 , we get

$$
\operatorname{deg}\left(\sum_{C \text { an equivalence class of } \sim} A^{\operatorname{sgn}(\sigma)}\left\langle\mathcal{S}_{\sigma}^{n}\right\rangle\right) \leq \operatorname{deg}\left(A^{\operatorname{sgn}(+)}\left\langle\overline{\mathcal{S}_{+}^{n}}\right\rangle\right)-4(n-1) .
$$

\section{Detecting Semi-ADeQuacy using the COlORED Jones POlynomial}

We use Theorem 1.1 to define a link invariant as in [KL14]. Let $D$ be a diagram of an oriented link $K$. Let $c_{-}(D)$ be the number of negative crossings of $D$, and recall that $\left|s_{+}(D)\right|$ is the number of state circles in the all +-resolution of $D, c(D)$ is the number of crossings in $D$, and $\omega(D)$ is the writhe of $D$. We consider the complexity

$$
\left(c_{-}(D), c(D),\left|s_{+}(D)\right|-\omega(D)\right),
$$

ordered lexicographically. Let $\mathcal{D}(K)$ be the set of diagrams of $K$ which minimizes this complexity. Recall that the lower bound $h_{n}(D)$ of the minimum degree $d(n)$ of $J_{K}^{n}(q)$, defined in (6), is $-\frac{1}{4} H_{n-1}(D)+\frac{(n-1)^{2}+2(n-1)}{4} \omega(D)$, where

$$
H_{n}(D)=n^{2} c(D)+2 n\left|s_{+}(D)\right| .
$$

Definition 4.1. Let $K$ be a link and $D$ an oriented link diagram in $\mathcal{D}(K)$. For $i \geq 3$, let $\beta_{i}=\beta_{i}(D)$ be the coefficient of $q^{h_{i}(D)+i-3}$ in $J_{K}^{i}(q)$. Define

$$
J_{D}^{+}(q):=\sum_{i=3}^{\infty} \beta_{i} q^{i} .
$$

We will need the following lemma from [KL14]. 
Lemma 4.2. KL14, Lemma 3.4] Suppose that for a link $K$, there is a diagram $D \in \mathcal{D}(K)$ that is +-adequate. Then, all the diagrams in $\mathcal{D}(K)$ are +-adequate.

Applying Theorem 1.1, we have the following corollaries.

Corollary 4.3. $J_{D}^{+}(q) \neq 0$ if and only if $D$ is +-adequate.

Proof. If $D$ is not +-adequate, then Theorem 1.1 says that $d(i) \geq h_{i}(D)+i-2$, so $\beta_{i}=0$ for all $i$. This shows the forward direction. For the converse, $\beta_{3}$ is the coefficient of $q^{h_{3}(D)}$ in $J_{K}^{3}(q)$. If $D$ is +-adequate, then $h_{3}(D)$ is equal to the minimum degree $d(3)$ of $J_{K}^{3}(q)$, so $\beta_{3} \neq 0$, and this shows that $J_{D}^{+}(q) \neq 0$.

Corollary 4.4. The power series $J_{D}^{+}(q)$ defined above is independent of the diagram $D \in \mathcal{D}(K)$, thus it is an invariant of $K$, which we denote by $J_{K}^{+}(q)$.

Proof. If $K$ is not +-adequate, then any diagram in $\mathcal{D}(K)$ is not +-adequate. Let $D$ be a diagram in $\mathcal{D}(K)$, then by Theorem 1.1. $J_{D}^{+}(q)=0$. If $K$ is +-adequate, then an +-adequate diagram of $K$ minimizes the complexity $\left(c_{-}(D), c(D),\left|s_{+}(D)\right|-w(D)\right)$, thus it belongs to $\mathcal{D}(K)$, and all the diagrams in $\mathcal{D}(K)$ are +-adequate by Lemma 4.2. Let $D$ be a diagram in $\mathcal{D}(K)$. As shown in Arm13], $J_{D}^{+}(q)$ records the stable coefficients of the sequence $\left\{J_{K}^{n}(q)\right\}_{n=2}^{\infty}$, therefore it is also independent of the diagram $D$.

Definition 4.5. Let $D$ be a link diagram. Consider the graph $s_{+}(D)$ with vertices the state circles, and edges the segments from the all-+ Kauffman state of $D$. We denote by $\chi_{+}(D)$ the Euler characteristic of $s_{+}(D)$.

Corollary 4.6. Suppose $D$ is an +-adequate diagram of a link $K$ and $D^{\prime}$ is another diagram of $K$. Then $D^{\prime}$ is +-adequate if and only if $c_{-}(D)=c_{-}\left(D^{\prime}\right)$ and $\chi_{+}(D)=\chi_{+}\left(D^{\prime}\right)$.

Proof. If $D^{\prime}$ is +-adequate, then $c_{-}(D)$ and $\left|s_{+}(D)\right|-w(D)$ are invariants of $K$ [Lic97, Theorem 5.13]. Thus, $\chi_{+}(D)=\left|s_{+}(D)\right|-c(D)=\left|s_{+}(D)\right|-w(D)-2 c_{-}(D)$ is also an invariant of $K$. For the converse, since $D$ is +-adequate, the minimum degree $d(n+1)$ of $J_{K}^{n+1}(q)$ is equal to $h_{n+1}(D)$ for all $n \geq 1$. We rewrite $h_{n+1}(D)$ here slightly differently:

$$
\begin{aligned}
h_{n+1}(D) & =-\frac{1}{4}\left(n^{2} c(D)+2 n\left|s_{+}(D)\right|-\omega(D)\left(n^{2}+2 n\right)\right) \\
& =-\frac{1}{4}\left(2 c_{-}(D) n^{2}+2 n\left(\left|s_{+}(D)\right|-\omega(D)\right)\right)
\end{aligned}
$$

If $D^{\prime}$ is not + -adequate but $c_{-}(D)=c_{-}\left(D^{\prime}\right)$ and $\chi_{+}(D)=\chi_{+}\left(D^{\prime}\right)$, then $\left|s_{+}(D)\right|-\omega(D)=$ $\left|s_{+}\left(D^{\prime}\right)\right|-\omega\left(D^{\prime}\right)$, so $h_{n+1}(D)=h_{n+1}\left(D^{\prime}\right)$. Theorem 1.1 applied to $D^{\prime}$ will imply that $d(n+1)<$ $h_{n+1}(D)$ for $n \geq 2$, which is a contradiction.

\section{REFERENCES}

[AD] Cody Armond and Oliver T. Dasbach, Rogers-Ramanujan type identities and the head and tail of the colored Jones polynomial, arXiv:1106.3948.

[AD17]_ The head and tail of the colored Jones polynomial for adequate knots, Proceedings of the American Mathematical Society 145 (2017), no. 3, 1357-1367.

[Arm13] Cody Armond, The head and tail conjecture for alternating knots., Algebr. Geom. Topol. 13 (2013), no. 5, 2809-2826.

[CK12] Benjamin Cooper and Vyacheslav Krushkal, Categorification of the Jones-Wenzl projectors, Quantum Topology 3 (2012), no. 2, 139-180.

[DL06] Oliver T. Dasbach and Xiao-Song Lin, On the head and the tail of the colored Jones polynomial, Compositio Mathematica 142 (2006), no. 5, 1332-1342.

[DL07] _ A volum-ish theorem for the Jones polynomial of alternating knots, Pacific J. Math. 231 (2007), no. 2, 279-291.

[FGL02] Charles Frohman, Razvan Gelca, and Walter Lofaro, The A-polynomial from the noncommutative viewpoint, Trans. Amer. Math. Soc. 354 (2002), no. 2, 735-747. 
[FKP08] David Futer, Efstratia Kalfagianni, and Jessica S. Purcell, Dehn filling, volume, and the Jones polynomial, J. Differential Geom. 78 (2008), no. 3, 429-464.

[FKP11] Slopes and colored Jones polynomials of adequate knots, Proc. Amer. Math. Soc. 139 (2011), no. $5,1889-1896$.

[FKP13] Guts of surfaces and the colored Jones polynomial, Lecture Notes in Mathematics, vol. 2069, Springer, Heidelberg, 2013.

[FKP14] _ Quasifuchsian state surfaces, Trans. Amer. Math. Soc. 366 (2014), 4323-4343.

[FKS06] Igor Frenkel, Mikhail Khovanov, and Catharina Stroppel, A categorification of finite-dimensional irreducible representations of quantum $\mathfrak{s l}_{2}$ and their tensor products, Selecta Mathematica. New Series 12 (2006), no. 3-4, 379-431.

[Gar11] Stavros Garoufalidis, The Jones slopes of a knot, Quantum Topology 2 (2011), 43-69.

[GL05] Stavros Garoufalidis and Thang T. Q. Lê, The colored Jones function is q-holonomic, Geom. Topology. (2005), no. 9, 1253-1293.

[GL15]_ N N N N _ sums, stability and the colored Jones polynomial, Research in Mathematical Sciences 2 (2015), 1-55.

[GNV16] Stavros Garoufalidis, Sergei Norin, and Thao Vong, Flag algebras and the stable coefficients of the Jones polynomial, European J. Combinatorics 51 (2016), 156-189.

[GV17] Stavros Garoufalidis and Thao Vong, A stability conjecture for the colored Jones polynomial, Topology Proceedings 49 (2017), 211-249.

[JCC14] C. Livingston J. C. Cha, Knotinfo: Table of knot invariants, 2014.

[Kal18] Efstratia Kalfagianni, A Jones slopes characterization of adequate knots, Indiana Univ. Mathematics Journal 67 (2018), no. 1, 205-219.

[Kas97] R. M. Kashaev, The hyperbolic volume of knots from the quantum dilogarithm, Lett. Math. Phys. 39 (1997), no. 3, 269-275.

[Kau87] Louis H. Kauffman, State models and the Jones polynomial, Topology 26 (1987), no. 3, 395-407.

[KL14] Efstratia Kalfagianni and Christine Ruey Shan Lee, On the degree of the colored Jones polynomial, Acta Mathematica Vietnamica (Proceedings of Quantum Topology and Hyperbolic Geometry in Nha Trang, May 2013) 39 (2014), no. 4, 549-560.

[Lee18] Christine Ruey Shan Lee, A trivial tail homology for non-A-adequate links, Algebr. Geom. Topol. 18 (2018), no. 3, 1481-1513.

[Lic97] William B. R. Lickorish, An introduction to knot theory, Graduate Texts in Mathematics, vol. 175, Springer-Verlag, New York, 1997.

[LT88] W. B. Raymond Lickorish and Morwen B. Thistlethwaite, Some links with nontrivial polynomials and their crossing-numbers, Comment. Math. Helv. 63 (1988), no. 4, 527-539.

[Man04] P. M. G. Manchón, Extreme coefficients of Jones polynomials and graph theory, J. Knot Theory Ramifications 13 (2004), no. 2, 277-295.

[MM01] Hitoshi Murakami and Jun Murakami, The colored Jones polynomials and the simplicial volume of a knot, Acta Math. 186 (2001), no. 1, 85-104.

$\left[\mathrm{MMO}^{+} 02\right]$ Hitoshi Murakami, Jun Murakami, Miyuki Okamoto, Toshie Takata, and Yoshiyuki Yokota, Kashaev's Conjecture and the Chern-Simons Invariants of Knots and Links, Experiment. Math. 11 (2002), no. 3, 427-435.

[Roz14a] Lev Rozansky, An infinite torus braid yields a categorified Jones-Wenzl projector, Fundamenta Mathematica 225 (2014), no. 1, 305-326.

[Roz14b] Khovanov homology of a unicolored B-adequate link has a tail, Quantum Topology 5 (2014), no. $4,541-579$.

[Sto11] Alexander Stoimenow, Coefficients and non-triviality of the Jones polynomial, Journal für die Reine und Angewandte Mathematik 657 (2011), 1-55.

[Wen87] Hans Wenzl, On sequences of projections, C.R. Math. Rep. Acad. Sci. Canada 9 (1987), no. 1, 5-9.

Department of Mathematics and Statistics, University of South Alabama, Mobile AL 36608

E-mail address: christine.rs.lee@gmail.com 